\title{
EVOLVING INFORMATION TECHNOLOGY: OPPORTUNITIES AND CHALLENGES
}

\author{
Ayub Hussein shirandula, Lilian Cherotich Ronoh, Kelvin Omieno \\ Masinde Muliro University of Science and Technology, Box 190 kakamega, \\ ayubshirandula@yahoo.com or ashirandula@mmust.ac.ke \\ Masinde Muliro University of Science and Technology, Box 190 kakamega, \\ ronomit@gmail.com or Irono@mmust.ac.ke \\ Masinde Muliro University of Science and Technology, Box 190 kakamega, \\ kelviomieno@gmail.com or komieno@mmust.ac.ke
}

\section{ABSTRACT}

The rapid Information technology evolution is fast becoming a change agent in the ways we conduct business and social interaction. Due to the increasing number of regulations and policies regarding information technology and also sustainable development, the issues of the cost, efficient, sharing, reuse and recycling matters have become the core challenges. As a consequence, this paper discusses the current physical infrastructure, broadband speed, and increasing bandwidth, that has lead to the transition of the television, radio broadcasting, and print media to digital technology convergence. The convergence of new technology is expected to continue evolving over the next decade, creating new opportunities and challenges. Location based services are expected to evolve through devices or objects with radio frequency identification (RFID) and Global Positioning System (GPS) connectivity. Traditional and new media are and will keep on increasing the social networking market due to the growth in online advertising revenue. Cloud computing will offer scale flexibility and cost efficiency. It will have the potential to be highly disruptive to desktop computing technology within the next few years. The paper highlights some opportunities, challenges and suggests some risks mitigation strategies.

\section{Indexing terms/Keywords}

Information technology, broadband, bandwidth, transition, Global Positioning System, networking, cloud computing

\section{Council for Innovative Research}

Peer Review Research Publishing System

\section{Journal: INTERNATIONAL JOURNAL OF COMPUTERS \& TECHNOLOGY}

Vol .14, No.8

www.ijctonline.com, editorijctonline@gmail.com 


\section{INTRODUCTION}

Information and communication technologies (ICT) over years have been undergoing through rapid revolution. The 'new' information technologies have a tendency to change things rapidly and fundamentally, so that society, industries, companies, countries, etc have undergone an enormous structural change. Data and information has been made available 24 hours. Technology has driven an information society free from traditional economic, political, and cultural constraints. Thus, according to (Toffler, 1978), 'the political technology of the Industrial Age, is no longer appropriate technology for the new civilization taking form around us. Recently, Google commercially launched Google Wallet, a cloud-based mobile payments ecosystem that promises to let users pay for things with their Android smart phones. Google Wallet replaces the act of pulling out a wallet, handing over a credit card, and signing your name, with pulling out your Smartphone, firing up an app, punching in a four-digit (personal identification number) PIN number, and tapping your phone against a payment reader. It is a nice opportunity but it takes less than 18 minutes for a hacker, or even someone with a fancy calculator, to guess a four-digit numeric PIN number matter what efforts Google and its partners have made to secure Google Wallet, PCMag's lead security analyst Neil Rubenking says the four-digit PIN authentication prevents him from trusting Google Wallet (Sara Y., 2011).

The effects of technology change are felt by all human beings. Every day people are reminded that they are living in the information age, in that they communicate through the internet, use phones, get immediate worldwide news, download music and movies, and buy airline tickets and book hotel rooms on line.

At the 2011 Black Hat Security Conference in August (Sara Y. 2011). According to Dai, if someone uses a brute-force attack, the method of simply going through different permutations of keys, it only takes: 18 minutes to crack a 4-digit numeric PIN, 51 hours to crack a 4-digit alphanumeric PIN, 8 years to crack a 6-digit alphanumeric PIN, 2 million years to crack an 8-digit alphanumeric-complex PIN. Complex characters are any other symbols that aren't letters or digits. Similarly, security expert Graham Cluley of Sophos Security (Sara Y. 2011) was concerned whether or not users would choose sensible PINs. In August 2011, Sophos found that $67 \%$ of consumers don't use a password to protect their mobile phones or laptops. And for those who do, they use the most common PIN numbers, as shown on the chart below.

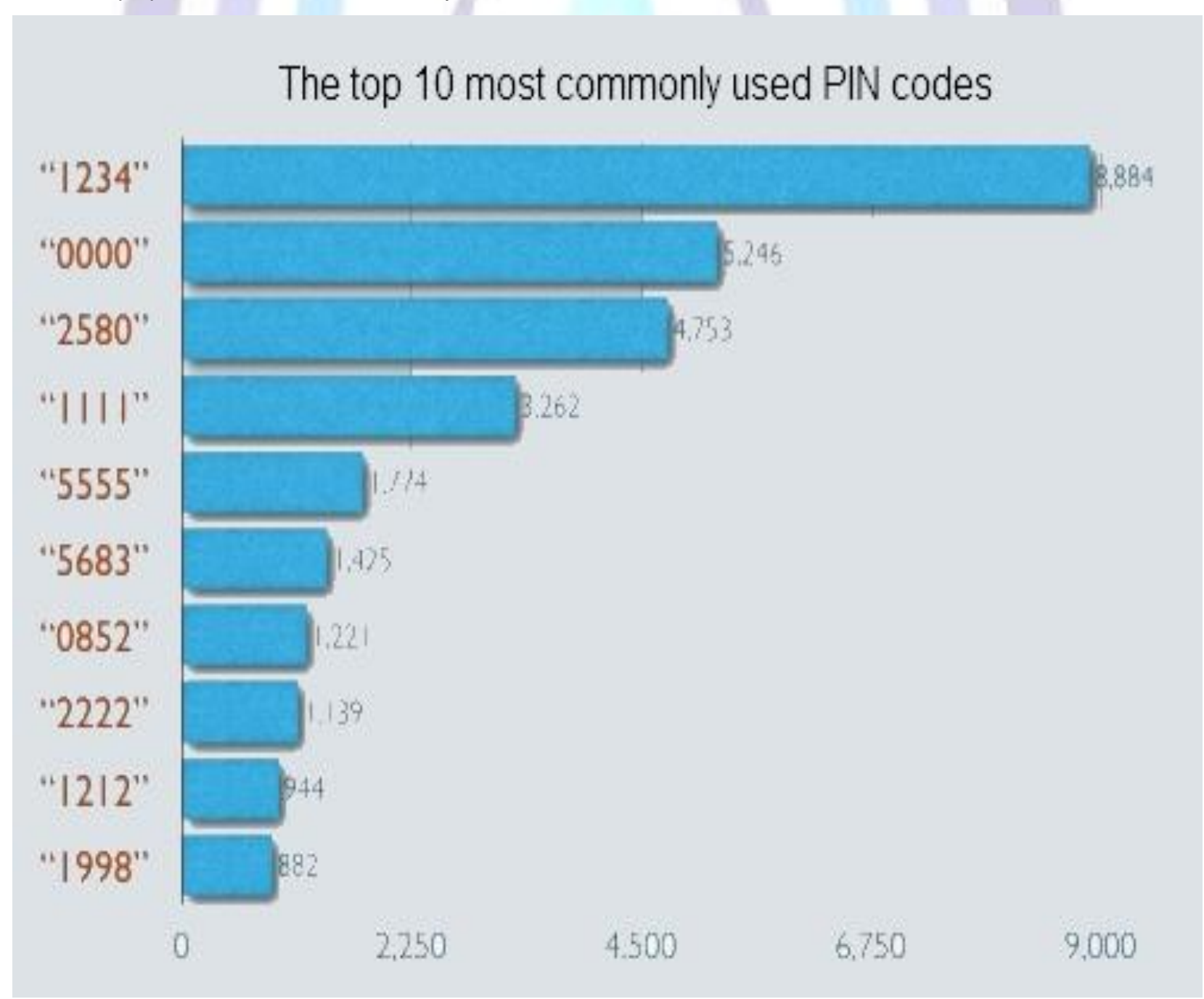

Fig 1: Top 10 most commonly used pin codes (source: http://www.pcmag.com) 
The new technology has revolutionized culture, business, entertainment, and research. The marriage of computers and telecommunications has lead to the growth of multimedia system of communication that has a global reach and at low cost, has brought about a fundamental transformation in the way humans communicate and interact. There is general consensus on the growing importance of ICT today. However, agreement is elusive when it comes to pin pointing the impact of this development on other issues such as security. In this paper, we discuss the 'information revolution' in relation to Innovations in technology, services, digitization, higher-speed broadband networks, diversity in physical infrastructure, distributed connectivity and the emerging social web.

\section{TECHNOLOGICAL CONVERGENCE}

Technology convergence is defined as the tendency for different technological systems to evolve towards performing similar tasks. Convergence can refer to previously separate technologies such as voice (and telephony features), data (and productivity applications), and video that now share resources and interact with each other synergistically.

The rise of digital communication in the late 20th century has made it possible for media organizations (or individuals) to deliver text, audio, and video material over the same wired, wireless, or fiber-optic connections. At the same time, it inspired some media organizations to explore multimedia delivery of information. This digital convergence of news media, in particular, was called "Mediamorphosis" as per researcher (Fidler 1997) Today, we are surrounded by a multi-level convergent media world where all modes of communication and information are continually reforming to adapt to the enduring demands of technologies, "changing the way we create, consume, learn and interact with each other".

\section{REVIEW OF THE PRESENT}

To understand today's technological trends; we need to look at how they have developed over the years. In education, computers have a long history. For example, the ENIAC (Electronic Numerical Integrator and Computer), built at the University of Pennsylvania's Moore school of Electrical Engineering between 1944 and 1946, was the first large scale general purpose electronic computer (Goldschmidt \& Akera, 1998). It weighed 30 tons, contained 19,000 vacuum tubes, 1,500 relays, and consumed almost 200 kilowatts of electrical power (Weik, 1961). Designed to calculate trajectory tables for new guns, the ENIAC failed on an average of every seven minutes, but when it worked it could compute 10 digit multiplication in $3 / 1000^{\text {th }}$ of second a huge accomplishment for its day (Jukes \& McCain, 1997). More recently, the 1980 model Cray supercomputer was the fastest machine of its day. It cost $\$ 12$ million, weighed five tons, and consumed 150 $\mathrm{KW}$ of electricity. It had only $8 \mathrm{MB}$ of RAM and operated at speed of $80 \mathrm{MHz}$ as per (Jukes \& McCain, 1997). A used computer today with the same capabilities can be purchased for under $\$ 300$. Since the popularization of the desktop computer in the 1980s, we have become painfully aware of how quickly computers become outdated. Many of today's educators point to this trend in their argument against the use of computers. This trend of increased power at lower cost is likely to continue well into the next century and has popularly become known as Moore's law, (Gordon 1965) Moore suggested that technology doubled in processing power approximately every 18 months and at the same time the price for that technology declined by about $35 \%$ a year relative to this power. The accuracy of Mr. Moore's prediction has proven to be frighteningly accurate. In a 1993 speech, Randall Tobias, put Moore's law in perspective when he said, " if we had had similar gains in automotive technology, today you could buy a luxus for about \$2. (Gordon Moore, 1964) It will travel at the speed of sound, and go 600 miles on a thimble of gas. It will be only three inches long but easy to parallel park".

Reflecting on changes that have occurred since 2006 highlights the sheer pace of change. Indicators of the trend towards pervasive computing are already evident in distributed, real-time data networks and networked high-resolution display screens, particularly in urban environments. Consumers are creating, re-using, remixing and distributing content one-tomany and one-to-one. When and where content is viewed, is subject to personal preferences. News is captured in digital form and distributed by individuals-more or less in real time. The Social Web has enabled people to connect and communicate in new ways, driving new forms of social interaction and entrepreneurship. At the same time, identifying and meeting the needs of people who may lack the capacity or desire to access and use new forms of communications and media is an emerging social inclusion issue.

\section{OPPORTUNITIES OF CONVERGENCE}

Appropriately managed Technological convergence in some countries, play an important role in economic, social, political, entertainment and governance. Such Governments are capitalizing on the opportunity to stimulate market development and meet previous unmet society communication needs.

Market Competition has increased because convergence has lowered barriers of entry to the market both for new operators and existing service providers. Thus consumers have been given a pool of providers and services to choose from hence the communication cost has been lowered. Industry boundaries are becoming blurred, allowing service providers to offer services in multiple markets. E.g. besides access to television, cable operators can also offer voice telephony and internet services.(Lee 2003)

Content providers can now easily access consumers with no need to own the distribution network. For example, a company may produce TV content and distributed it through cable networks without having to own it. 

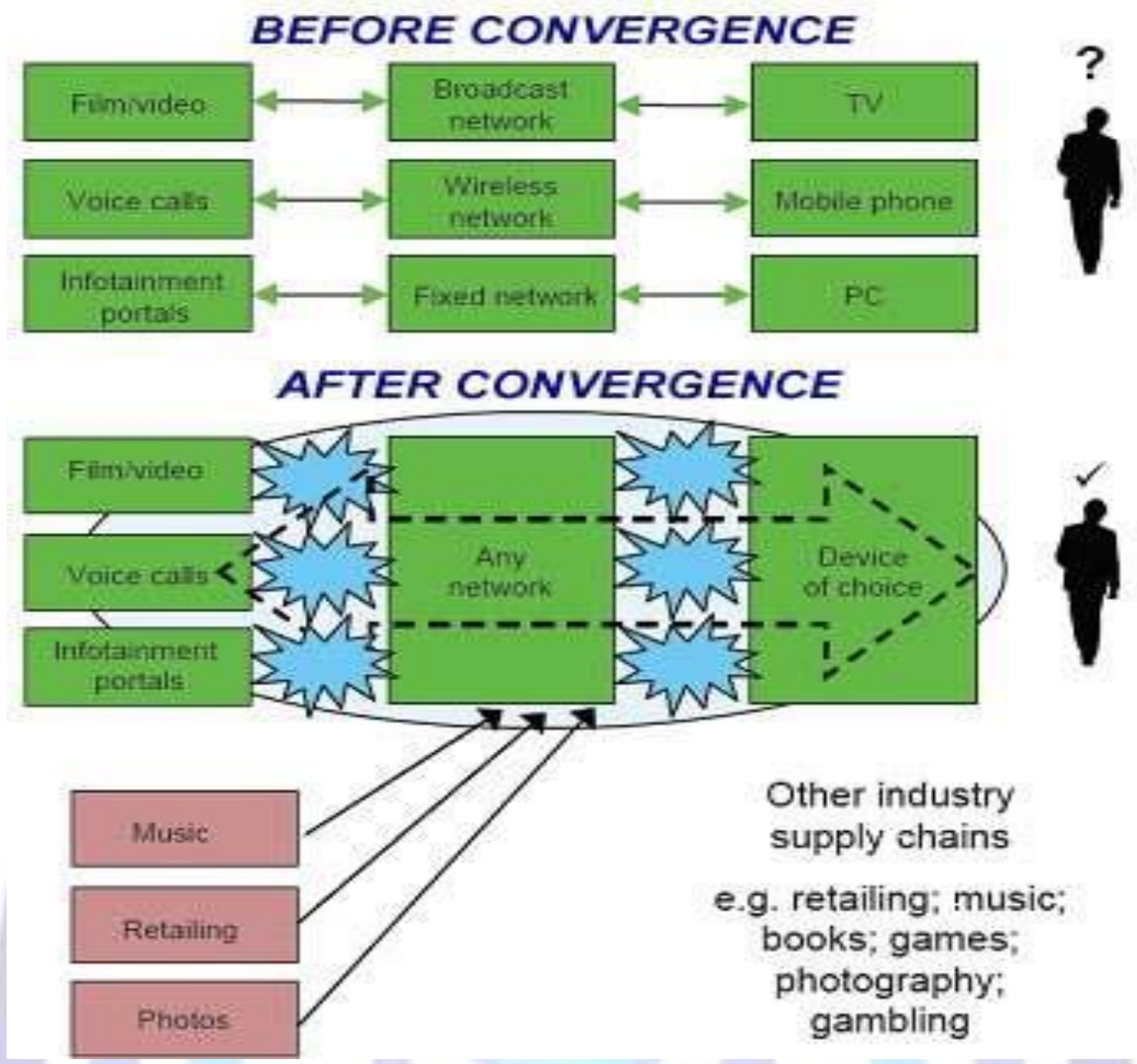

Fig 2: Source: (HP, USTTI Training 2006) ICT industry before and after technological convergence

From the diagram above First, it allow for user mobility by untethering users from fixed Communications stations such as desktop PCs or landline telephones. Second, one can achieve broad spatial coverage with less deployment effort due to the reduced need for installing cables.

\section{NEW SERVICES AND APPLICATIONS}

Established companies have found in convergence opportunities to operate more efficiently, most of them have increased their returns on investing in technology and are realizing other business benefits through development of new services and rapid market expansion. Convergence has opened up new sales markets for companies, a case observed in mobile operators e.g safaricom, and Airtel as the market saturates, they are branching into non-voice services, such as video streaming, portals, messaging, M-PESA, and gaming, to drive future revenue growth. New applications have given rise to new ways of entertainment (i.e. online gaming) and socialization (i.e. chat rooms, face book, twitter e.tc). The convergence of voice, video and data has given consumers new ways of communication as they can all talk, send text and video over one single network, using one single application at much lower costs than before.

(QuantCast, 2007) Social networking sites (SNS) may evolve over the next five years to become integrated hubs for individuals, organisations and their extended networks to connect, communicate, access and share tailored news, information and entertainment. They are becoming social destinations in their own right, which can lead to a 'blurring of the boundaries' between online and offline worlds. Young people in particular have been quick to use SNS to share information about them, meet their need for self-expression, make new connections and organise social activities. Some organisations see real-time collaboration through social networking as an effective way to manage large-scale and geographically dispersed teams, and for organisational communication.

\section{CONVENIENCE AND SIMPLICITY}

Currently consumers find in convergence an opportunity to enjoy the convenience of having many devices all in one, saving on both size and ownership costs. For example, a single mobile phone device can receive television programmes and play videos, thus enabling simplicity and convenience in device ownership as one device can be used to access multiple services. E.g airport hotel directory on the web now offers hotel-booking services on the iPhone and all Internetwork Operating System (los) devices. . (Tony E. 2011) 
The app uses state of the art technology and offers an upgraded hotel inventory without losing sight of Airport Hotel Guide's primary goal of providing affordable and targeted hotel accommodations. The Airport Hotel Guide app unlocks 200,000 hotels near 37,000 airports and cities worldwide, providing hotel solutions to any kind of traveler and travel need. (Tony E. 2011)

The web has enabled highly innovative developments at the edge. The web is now turning into both a platform and a database. Recent examples of this include the third-party development of applications programming interface (APIs), enabling interaction between programs on networked computers.

\section{INTELLIGENT TRANSPORT SYSTEMS TECHNOLOGIES}

Intelligent Transportation Systems (ITS) use a broad range of wireless technologies that, when integrated into the transportation system infrastructure and vehicles themselves, help to monitor and manage traffic flow, relieve congestion, provide alternative routes to travellers, improve safety and save lives. The ITS technologies are enabled using Dedicated Short Range Communications (DSRC).

\section{LOCATION-SENSING AND CONTEXT-AWARE TECHNOLOGIES}

Today wireless devices know with increasing accuracy where a user is. Location-based services are expected to evolve through devices or objects with (Radio-frequency identification) RFID and GPS connectivity. The Geographic Information System (GIS) is becoming more widely deployed for tasks such as requesting directions, locating services or directing emergency service response. Examples of location services are mobile phones delivering location-based marketing and real-time traffic information, monitoring local, interstate and international delivery vehicles, monitoring limited-release prisoners, buddy finder services and directory-style information. They also encompass GPS functionality in mobile phones, digital cameras and real-time maps to hand-held devices and cars. (George R. 2011)

The ability to track users' location, has improved even though it has created considerable privacy concerns.

\section{CLOUD COMPUTING}

Also known as 'computing on demand' or 'software as a service', cloud computing is the shared use of distributed computing resources as an alternative to in-house IT applications and services using local servers or personal devices. Cloud computing offers scale flexibility and cost-efficiency, and has the potential to be highly disruptive to desktop computing technology within the next five years.

There are some non-trivial challenges involved in adopting cloud computing that must be watched closely. These includes a long list of issues such as the security and privacy of business data in remote 3rd party data centers, the dreaded concerns about platform lock-in, worries about reliability/performance, and even fears about making the wrong decision before the industry begins to mature. (Goodman, B. D. 2010)

However, in a business environment where change is inevitable in order to survive, cloud computing appears to offer significant economic benefits if the risks can be offset. Hence, one of the bigger challenges that IT departments will face is whether they can take the plunge with cloud computing quickly enough to benefit their organizations as a whole. (Zhou, R. S 2010)

\section{BROADBAND}

Convergence has given rise to new services and applications which are bandwidth intensive, which require an existence of broadband infrastructure. Only with broadband access is the use of complex services for example multimedia services attractive or possible in the first place. While developed economies may not face a bandwidth shortage dilemma, the same may not be said about most of the developing economies such as Kenya, whose telecommunication infrastructures are based on narrowband technologies. These countries face challenges of having to upgrade their infrastructure or miss on the benefits of the technological convergence. In meeting this challenge, as it was in the past, financial constraints have continued to be a problem.

"Building High Speed Broadband Networks extends the performance of our Physical Infrastructure and hence is an added investment in Infrastructure" as per (Owen McShane, 2008) Director of the Centre for Resource Management Studies .

Higher bandwidth is necessary to meet demand for increasingly data-rich and multimedia traffic that is increasingly twoway; that is, upstream and downstream. Wired local access data rates of up to $30 \mathrm{Mbit} / \mathrm{s}$ are available in some areas. The highest average broadband speed internationally is currently in Japan, where optic-fibre deployment provides local access rates nearing $100 \mathrm{Mbit} / \mathrm{s}$. In terms of wide-area networks, a new standard scalable beyond $10 \mathrm{Gbit} / \mathrm{s}$ using optic-fibre technology is under development. The deployment of multi-Gigabit data rates over optic-fibre networks in Australia is technically possible over the next few years. (Griffins, 2008).

Many Governments are committed to delivering a national high-speed broadband network. E.g In 2008 an Australian government had announced plans for mobile network operator to deliver local access speeds in excess of $40 \mathrm{Mbit} / \mathrm{s}$ by 2009 using HSPA+ technology. Some expected that by 2012, this mobile network will be capable of theoretical speeds of $100 \mathrm{Mbit} / \mathrm{s}$. Australian researchers are working on multi-Gigabit wireless data rates using millimetre wave spectrum. The 
deployment of such networks in the world to complement other local area networks and for ad hoc communications may be possible within the next few years (Griffins, 2008).

We are having radical departure from the tradition of putting machines out for our use, and having us adapt to them. Instead, in the world of ubiquitous computing, technology will be implicit in our lives, built in to the things we use, including the spaces. The proponents of this technology hold that this type of computing will be a more natural tool, and thus a more powerful and effective one for us to use.

\section{DIGITAL BROADCASTING}

TV and radio broadcasting are in transition to digitisation. Digital terrestrial broadcasting also competes with digital broadcasting via satellite and cable. In overseas markets where sufficient broadband bandwidth is being deployed (6 Mbits/s seems to be a benchmark), there is competitive internet delivery of broadcast-like services. In Australia, the market for Internet Protocol Television (IPTV) services is at an early stage of development. In a globally interconnected market, local content will compete with directly delivered international digital content. (Gustavo G. 2007)

Mobile distribution of video content is developing as a complementary platform to viewing digital content on the television set and PC.

\section{COGNITIVE AND SOFTWARE-DEFINED RADIO}

Some smart radio capabilities are available now on the market, cognitive radio (CR) and software-defined radio (SDR) technologies are frequency agile and share spectrum without interference. Ideally, CR can individually and dynamically adapt its spectrum usage, taking into account local RF conditions such as interference and demand (the number of users and the applications they are using). Peter S. (2009)

SDR-operating parameters are defined by its software rather than its hardware, enabling it to tune into different frequencies and receive any modulation across a large frequency spectrum. By its design, the SDR can receive and transmit different forms of radio protocols simply by running different software. SDR enables fast upgrades and extremely flexible operation. Peter S. (2009)

Software-defined mobile phone base station is able to use a software upgrade rather than an expensive hardware upgrade to implement significant changes to its network, subject to the limitations of radio-frequency input/output elements. Currently a software-defined handset can be upgraded 'over-the-air' to operate on the new network without the customer having to replace the handset. The many economic and technical advantages of SDR mean that its use will continue to grow.

\section{EFFICIENCY TECHNIQUES IN MULTIMEDIA TRANSMISSION}

The amounts of data that can be transmitted over a given physical infrastructure are increasing due to the ongoing technological developments. We have relentless research and development in compression technologies that is expected to better handle bandwidth. (Allen G.1994)

The combination of advancements in encoding and compression techniques continues to deliver large increases in the effective capacity of existing infrastructure, and also contributes to new or innovative methods of communication. These developments have important economic implications for the carriage aspects of the industry and will make bandwidthhungry services available sooner, hastening convergence. (Laurent D. 1999)

\section{CHALLENGES AND MITGATION IN CONVERGENT WORLD}

As the internet and associated applications such as email and web browsers are used for an ever-increasing number of business and social transactions, organised crime is focusing increasingly on internet-related crime. (Phil W. 2002) One indicator of the growing economic importance of the internet is that the number of Australian internet banking users aged 16 years and older increased from 6.9 million in May 2006 to 8.2 million in May 2007.

Given the burgeoning online economy and increasingly sophisticated criminal incursions into this economy, particularly through the use of botnets, maintaining and enhancing the security of internet transactions and communications will become a growing priority. Anti-botnet measures will be increasingly important, given the role of botnets in stealing personal identity information, disseminating malware (malicious software) through spam and mounting attacks on internet websites through distributed denial of service attacks. (Shun-Yung K.W \& Wilson H. 2011)

We have a growing focus on website security to prevent legitimate websites from becoming unwitting hosts of malware. Social networking sites are likely to be prime targets of criminal attacks, as are other websites with significant potential for the harvesting of personal identity information, including government websites.

Improved authentication and validation systems for internet communication and transactions need to be developed, both for high-level transactions such as internet banking and for more routine communications such as email, M_PESA e.t.c.

The international governance of the internet will be an expanding area of focus, particularly in areas such as domain management and assignment. Obtaining a balance between maintaining individual privacy and effective e-security arrangements will be a major challenge to these governance arrangements. 


\section{CONCLUSION}

Convergence, in usual sense, means coming together. In the world of technology, it is the major communications platforms (broadcasting, telecommunications and online) that are coming together so that their once separate functions now overlap. Video content, for example, that used to be available only on television can be viewed easily over the internet. A person can now watch exactly the same TV program on a TV set, laptop, or mobile phone. However, the underlying networks that are used to transmit the program are very different-broadcast spectrum or cable networks, the internet or mobile networks offers massive opportunities for the development of new value-added, Regardless of issues of adjustment to new environment, service providers, regulators and users. Services, efficiency, and expansions of market and users choice have increased.

\section{ACKNOWLEDGMENTS}

My thanks to Dr. Mbugua who have contributed towards development of the paper.

\section{REFERENCES}

[1] Allen G. 1994 Advances in speech and audio compression

[2] Dutton, William H. 1999, Society on the Line: Information Politics in the Digital Age.

[3] Donna L. Hoffman, Thomas P. Novak, and Marcos Peralta. 1999 Building consumer trust online. Communications of the ACM.

[4] Ryan Henry and Edward Peartree 1998, the Information Revolution and International Security.

[5] Graham, D. 1997. The classroom computer revolution that hasn't happened yet! Education Canada

[6] Goodman, B. D.2010 U.S. Patent Filed. "Linking Instances Within a Cloud Computing Environment"

[7] George R.2011 Location Sensing Technologies and Applications

[8] Griffins, p.2008, may 23. Reactions to broadband funding. Retrieved October 15 , 2011, from www.kiwiblog.co.nz

[9] Gustavo G.2007 Digital Television and Radio: Democratisation or greater concentration

[10] Laurent D. 1999 Advances in Digital Image Compression by Adaptive Thinning

[11] Jukes, I, \& McCain, T. 1997. Living on the future edge. The Thornburg Centre (online).

[12] Available: Http:// tcpd.org/tcpd/handouts.html October 1, 1998.

[13] Michael B.2000: A location-based remembrance appliance. Personal Technologies.

[14] M. Weiser, 1998 "The Future of Ubiquitous Computing on Campus".

[15] Molitor, G. 1998. Trends and forecasts for the new millennium. Futurist, 31(1). 53-59

[16] Mueller, M.,199 "Digital Convergence and its Consequences," Javnost-the Public.

[17] Peter S. 2009 Future Directions in Cognitive Radio Network Research

[18] Phil W. 2002 Organized Crime and Cyber-Crime: Implications for Business

[19] Shun-Yung K.W \& Wilson H.2011 The evolutional view of the types of identity thefts and online frauds in the era of the internet

[20] Tony E. 2011 Newly Designed Travel App Brings Convenience and Simplicity to the Hotel Booking Process.

[21] Vince, J.1999 Digital Convergence the Information Revolution, London: Springer.

[22] Wager, w. 1993. Tomorrow and tomorrow. Technology review 96, 52

[23] Wayner, P 2008, 'Cloud versus cloud: A guided tour of Amazon, Google, AppNexus, and

[24] GoGrid' - available at http://www.infoworld.com/d/cloud-computing/cloud-versus-cloudguided-

[25] tour-amazon-google-appnexus-and-gogrid-122

[26] Yoffie, D.B.,1996 "Competing in the Age of Digital Convergence", California Management Review.

[27] Zhou, R. S2010 ., "Bridging to the Cloud: Solution design trends helping "legacy" systems leverage cloud computing," Workflow Management in Service and Cloud Computing (WMSC2010), 2010 2nd International Workshop. 


\section{Author' biography with Photo}

\section{AYUB HUSSEIN SHIRANDULA}

Masinde Muliro Univ. of Sc. \& Tech P.O. Box 190, Kakamega,

Marital status: Married

Nationality: Kenyan

Date of birth: $1^{\text {st }}$ January, 1981 (AGE: 34 years)

Religion: Muslim

Languages spoken: English, Kiswahili

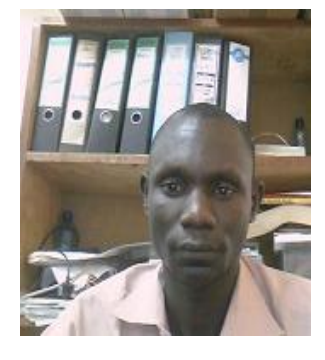

Mobile :0726940558

E-mail: ayubshirandula@yahoo.com or ashirandula@mmust.ac.ke

\section{LILIAN CHEROTICH RONOH}

Masinde Muliro Univ. of Sc. \& Tech P.O. Box 190, Kakamega,

Marital status: Married

Nationality: Kenyan

Date of birth: $1^{\text {st }}$ January, 1978 (AGE: 37 years)

Religion: Christian

Languages spoken: English, Kiswahili

Mobile :0720574243

E-mail: ronomit@gmail.com or Irono@mmust.ac.ke

\section{KELVIN K. OMIENO}

Masinde Muliro Univ. of Sc. \& Tech P.O. Box 190, Kakamega,

Marital status: Married

Nationality: Kenyan

Date of birth: $12^{\text {th }}$ march, 1984

Religion: Christian

Languages spoken: English, Kiswahili

Mobile :0726849197

E-mail: komieno@mmust.ac.ke 